\title{
Solutions to the Puzzles of Quantum Gravity and General Theory of Quantum Gravity and Quantum Gravity of the Universe and General Black Hole
}

\author{
C. Huang ${ }^{1,2}{ }^{*}$ Yong-Chang Huang ${ }^{3},^{\dagger}$ and Xinfei $\mathrm{Li}^{3, \ddagger}$ \\ ${ }^{1}$ Lawrence Berkeley National Laboratory, \\ 1 Cyclotron Road, Berkeley CA 94720, USA \\ ${ }^{2}$ Department of Physics and Astronomy, \\ Purdue University, 525 Northwestern Avenue, \\ W.Lafayette, IN 47907-2036, USA \\ ${ }^{3}$ Institute of Theoretical Physics, \\ Beijing University of Technology, Beijing 100124, China
}

(Dated: July 8, 2021)

\begin{abstract}
This paper gives both the solutions to the puzzles of quantum gravity and a general theory of quantum gravity, further shows quantum gravity of the Universe and general black hole, and discovers their relations reflecting symmetric propertis of the standard nonlinear gravitational Lagrangian, which are not relevant to any concrete metric models. This paper concretely shows the general commutation relations of the general gravitational field operators and their zeroth, first, second and third style, respectively, of high order canonical momentum operators for the general nonlinear system of the standard gravitational Lagrangian, and then has finished all the four styles of the quantization of the standard gravity. No needing, as usual, to solve the Euler-Lagrange equation to complete the whole process of the quantization of the standard gravitational fields, namely, this paper novelly simplifies all the current quantization theories of the standard gravitational fields. So lots of the complex calculations of quantum gravitational field theories up to now can be omitted to make the physical picture clearer, simpler and more easily understanding. Therefore, the solutions to puzzles of quantum gravity are given. Consequently, this paper opens a door to study and give a general theory of the quantum gravitational field don't depending on any concrete metric models.

Key words: general relativity, Lagrangian, operators, quantum gravity, quantization, commutation relation, the Universe, general black hole
\end{abstract}

PACS numbers: 04.90.+e

\section{INTRODUCTION}

Quantum gravity is a domain of theoretical physics for exploring to describe gravity on the basis of principles of quantum mechanics [1], when near compact astrophysical objects, there are the strong effects of gravity.

The current gravitational theory is based on Einsteinian general relativity in classical physics, and the other three physical fundamental forces are represented in quantum field theory, they are very different formalisms for explaining different physical phenomena [2]. Quantum gravity is necessary when people's studies are from classical physics to quantum physics [3-6].

Quantum gravity may reconcile general relativity with quantum mechanics, there exist difficulties when quantum field theory is used to gravitational theory by graviton bosons [7], and the deduced theory is not renormalizable (e.g., the theory shows infinite values of observable quantities, for instance, the masses of particles). Therefore, theorists have done a lot of research works in order to overcome the problems of quantum gravity. Superstring theory unifying gravity with the other three fundamental forces and loop quantum gravity no such attempt are the good candidates for overcoming the problems of quantum gravity [8], and both superstring theory and loop quantum gravity all quantize the gravitational field-
S.

Quantum gravity shows the quantum behavior of the gravitational field, and gravitational quantum field theory of unifying a grand unified theory may be viewed as a theory of everything. The investigations of quantum gravity are domains having different approaches for the unification.

Up to now, no less, at least, than 16 major interesting approaches for quantum gravity have been shown in the literature [9] in alphabetical order as follows:

Affine quantum gravity [10]; Asymptotic quantization $[11,12]$; Canonical quantum gravity [13-16]; Condensedmatter view [17]; Manifestly covariant quantization [1824]; Euclidean quantum gravity [25, 26]; Lattice formulation [27, 28]; Loop space representation [29, 30]; Noncommutative geometry [31]; Quantum topology [32], [33]; Renormalization group and asymptotic safety [34, 35]; Rsquared gravity [36]; String and brane theory [37-40]; Supergravity [41, 42]; Triangulations [43-45] and null-strut calculus [46]; Twistor theory [47, 48].

Quantum gravitational effects evidently show at scales near the Planck scale and equivalently far larger energy, which is far larger than that of current high energy particle accelerators. Consequently, there are not experimental data distinguishing the proposed competing theories, but thought experimental methods are presented as the 
testing methods of the competing theories [49-51].

The quantization of gravity, up to now, remains a formidable problem for physicists. Although superstring theory has made some progress in quantizing gravity, many profound questions still remain unanswered [52].

Meshing all these theories at all energy scales is relevant to the different assumptions how the universe works. General relativity shows that "spacetime tells matter how to move; matter tells spacetime how to curve." [53]. Quantum field theory is formulated according to special relativity in the flat spacetime. When treating gravitation as a simple quantum field, which will result in that the theory is not renormalizable [7]. Quantizing gravity becomes key challenges, and is no longer applicable in flat spacetime [54].

Quantum gravitational theory is widely hoped to understand the origin of the universe and the behaviors of black holes [55]. Some different quantum physics systems are investigated [56], and Minimal area surfaces dual to Wilson loops are studied [57].

The appearance of singularity of infinite large of spacetime curvature in general relativity (meaning its structure has a microscopic scale) requires the establishment of a complete theory of quantum gravity. The quantum gravitational theory needs to be able to describe the conditions inside black holes and in the very early universe, where gravity and the related spacetime geometry need to be described in quantized formulism. Despite lots of efforts by physicists and the developments of some potential candidate theories, humans have yet to come up with a complete and self-consistent theory of quantum gravity. This paper wants to solve the problems in order to give a complete and self-consistent general theory of quantum gravity.

The arrangement of this paper is: Sect. 2 gives canonical conjugate momentum opretors corresponding gravitational field operators, Sect. 3 studies energy of gravitational field of the standard gravtational Lagrangian, Sect.4 shows further investigations of quantization of quantum gravitational fields, Sect. 5 investigates commutation relation for gravitational fields and the first style of momenta, Sect. 6 shows commutation relation for gravitational fields and the second style of momenta, Sect. 7 studies commutation relation for graviational fields and the third style of momenta, Sect. 8 gives quantum gravity of the Universe and general black hole, and Sect. 9 is summary and conclusion.

\section{CANONICAL CONJUGATE MOMENTUM OPRETORS CORRESPONDING GRAVITATIONAL FIELD OPERATORS}

We generally consider granvitational field operators

$$
\hat{g}_{\mu \nu}(x)=\hat{g}_{\nu \mu}(x), \mu, \nu=0,1,2,3,
$$

thus there are ten independent components.

We need to specially stress that our investigations in this paper are very general for the standard gravitational field Lagrangian that has gotten the huge successes in classical physics, and are not dependent on any concrete metric model through the whole paper. Thus, the studies of this paper is of the general theory of quantum gravity.

When we generalize coordinate operators and canonical momentum operators of finite degrees of freedom to gravity field operators and its canonical conjugate momenta of infinite degrees of freedom, physics consistence requires us to generalize commutation relation of coordinate operators and momentum operators in the first quantization to commutation relation of gravity field operators and its canonical conjugate momenta

$$
\left[\hat{g}_{\mu \nu}(\mathbf{x}, t), \hat{\pi}^{\alpha \beta}\left(\mathbf{x}^{\prime}, t^{\prime}\right)\right]_{t=t^{\prime}}=i \Delta_{\mu \nu}^{\alpha \beta} \delta\left(\mathbf{x}-\mathbf{x}^{\prime}\right),
$$

where $\Delta_{\mu \nu}^{\alpha \beta}$ is a general operator function decided by satisfying some conditions of this system, the corresponding canonical momentum is

$$
\begin{gathered}
\pi^{\mu \nu}(\mathbf{x}, t)=\frac{\partial \mathcal{L}}{\partial \partial_{t} g_{\mu \nu}(\mathbf{x}, t)} \rightarrow \hat{\pi}^{\mu \nu}(\mathbf{x}, t)=\frac{\partial \hat{\mathcal{L}}}{\partial \partial_{t} \hat{g}_{\mu \nu}(\mathbf{x}, t)} \\
\mathcal{L}=\kappa R=\kappa g^{\alpha \beta} R_{\alpha \beta},
\end{gathered}
$$

where $\mathcal{L}, R$ and $\kappa$ are the standard Lagrangian density of general relativity [58], the scalar curvature and the coupling constant of the general gravity system, respectively.

Generally taking the standard Lagrangian density (2.4) of this system and using eq.(2.3), we get

$$
\pi^{\mu \nu}(\mathbf{x}, t)=\frac{\partial \mathcal{L}}{\partial \partial_{t} g_{\mu \nu}(\mathbf{x}, t)}=\kappa g^{\alpha \beta} \frac{\partial R_{\alpha \beta}}{\partial \partial_{t} g_{\mu \nu}(\mathbf{x}, t)} .
$$

Putting

$$
R_{\alpha \beta}=\Gamma_{\alpha \sigma, \beta}^{\sigma}-\Gamma_{\alpha \beta, \sigma}^{\sigma}+\Gamma_{\rho \beta}^{\sigma} \Gamma_{\alpha \sigma}^{\rho}-\Gamma_{\rho \sigma}^{\sigma} \Gamma_{\alpha \beta}^{\rho}
$$

into eq.(2.5), it follows that

$\pi^{\mu \nu}(\mathbf{x}, t)=\kappa g^{\alpha \beta} \frac{\partial\left(\Gamma_{\alpha \sigma, \beta}^{\sigma}-\Gamma_{\alpha \beta, \sigma}^{\sigma}+\Gamma_{\rho \beta}^{\sigma} \Gamma_{\alpha \sigma}^{\rho}-\Gamma_{\rho \sigma}^{\sigma} \Gamma_{\alpha \beta}^{\rho}\right)}{\partial \partial_{t} g_{\mu \nu}(\mathbf{x}, t)}$.

Using connection

$$
\Gamma_{\rho \beta}^{\sigma}=\frac{1}{2} g^{\sigma \gamma}\left(g_{\gamma \beta, \rho}+g_{\rho \gamma, \beta}-g_{\rho \beta, \gamma}\right),
$$


eq.(2.7) can be rewritten as

$$
\begin{gathered}
\pi^{t \mu \nu}(\mathbf{x}, t)=\kappa g^{\alpha \beta} \frac{\partial}{\partial \partial_{t} g_{\mu \nu}(\mathbf{x}, t)}[ \\
\frac{1}{2} g_{, \beta}^{\sigma \gamma}\left(g_{\gamma \sigma, \alpha}+g_{\alpha \gamma, \sigma}-g_{\alpha \sigma, \gamma}\right)-\frac{1}{2} g_{, \sigma}^{\sigma \gamma}\left(g_{\gamma \beta, \alpha}+g_{\alpha \gamma, \beta}-g_{\alpha \beta, \gamma}\right) \\
+\frac{1}{2} g^{\sigma \gamma}\left(g_{\gamma \beta, \rho}+g_{\rho \gamma, \beta}-g_{\rho \beta, \gamma}\right) \frac{1}{2} g^{\rho \gamma}\left(g_{\gamma \sigma, \alpha}+g_{\alpha \gamma, \sigma}-g_{\alpha \sigma, \gamma}\right) \\
\left.-\frac{1}{2} g^{\sigma \gamma}\left(g_{\gamma \sigma, \rho}+g_{\rho \gamma, \sigma}-g_{\rho \sigma, \gamma}\right) \frac{1}{2} g^{\rho \gamma}\left(g_{\gamma \beta, \alpha}+g_{\alpha \gamma, \beta}-g_{\alpha \beta, \gamma}\right)\right] .
\end{gathered}
$$

Using formulae

$g^{\sigma \mu} g_{\mu \beta, \rho}=-g_{, \rho}^{\sigma \mu} g_{\mu \beta}, g^{\sigma \mu} g_{\mu \beta, \rho} g^{\beta \alpha}=-g_{, \rho}^{\sigma \mu} g_{\mu \beta} g^{\beta \alpha}=-g_{, \rho}^{\sigma \alpha}$,

eq.(2.9) can be reexpressed as

$$
\begin{aligned}
& \pi^{t \mu \nu}(\mathbf{x}, t)=\kappa g^{\alpha \beta}\left[\frac{-1}{2} g^{\sigma \tau} \delta_{\beta}^{t} \delta_{\tau}^{\mu} \delta_{\varepsilon}^{\nu} g^{\varepsilon \gamma}\left(g_{\gamma \sigma, \alpha}+g_{\alpha \gamma, \sigma}-g_{\alpha \sigma, \gamma}\right)\right. \\
& \quad-\frac{1}{2} g^{\sigma \tau} g_{\tau \varepsilon, \beta} g^{\varepsilon \gamma}\left(\delta_{\alpha}^{t} \delta_{\gamma}^{\mu} \delta_{\sigma}^{\nu}+\delta_{\sigma}^{t} \delta_{\alpha}^{\mu} \delta_{\gamma}^{\nu}-\delta_{\gamma}^{t} \delta_{\alpha}^{\mu} \delta_{\sigma}^{\nu}\right) \\
& \quad+\frac{1}{2} g^{\sigma \tau} \delta_{\sigma}^{t} \delta_{\tau}^{\mu} \delta_{\varepsilon}^{\nu} g^{\varepsilon \gamma}\left(g_{\gamma \beta, \alpha}+g_{\alpha \gamma, \beta}-g_{\alpha \beta, \gamma}\right) \\
& +\frac{1}{2} g^{\sigma \tau} g_{\tau \varepsilon, \sigma} g^{\varepsilon \gamma}\left(\delta_{\alpha}^{t} \delta_{\gamma}^{\mu} \delta_{\beta}^{\nu}+\delta_{\beta}^{t} \delta_{\alpha}^{\mu} \delta_{\gamma}^{\nu}-\delta_{\gamma}^{t} \delta_{\alpha}^{\mu} \delta_{\beta}^{\nu}\right)+\frac{1}{4} g^{\sigma \gamma}\left(\delta_{\rho}^{t} \delta_{\gamma}^{\mu} \delta_{\beta}^{\nu}\right. \\
& \left.+\delta_{\beta}^{t} \delta_{\rho}^{\mu} \delta_{\gamma}^{\nu}-\delta_{\gamma}^{t} \delta_{\rho}^{\mu} \delta_{\beta}^{\nu}\right) g^{\rho \gamma^{\prime}}\left(g_{\gamma^{\prime} \sigma, \alpha}+g_{\alpha \gamma^{\prime}, \sigma}-g_{\alpha \sigma, \gamma^{\prime}}\right)+\frac{1}{4} g^{\sigma \gamma}( \\
& \left.g_{\gamma \beta, \rho}+g_{\rho \gamma, \beta}-g_{\rho \beta, \gamma}\right) g^{\rho \gamma^{\prime}}\left(\delta_{\alpha}^{t} \delta_{\gamma^{\prime}}^{\mu} \delta_{\sigma}^{\nu}+\delta_{\sigma}^{t} \delta_{\alpha}^{\mu} \delta_{\gamma^{\prime}}^{\nu}-\delta_{\gamma^{\prime}}^{t} \delta_{\alpha}^{\mu} \delta_{\sigma}^{\nu}\right)-
\end{aligned}
$$$$
\frac{1}{4} g^{\sigma \gamma}\left(\delta_{\rho}^{t} \delta_{\gamma}^{\mu} \delta_{\sigma}^{\nu}+\delta_{\sigma}^{t} \delta_{\rho}^{\mu} \delta_{\gamma}^{\nu}-\delta_{\gamma}^{t} \delta_{\rho}^{\mu} \delta_{\sigma}^{\nu}\right) g^{\rho \gamma^{\prime}}\left(g_{\gamma^{\prime} \beta, \alpha}+g_{\alpha \gamma^{\prime}, \beta}-g_{\alpha \beta, \gamma^{\prime}}\right)
$$$$
\left.-\frac{1}{4} g^{\sigma \gamma}\left(g_{\gamma \sigma, \rho}+g_{\rho \gamma, \sigma}-g_{\rho \sigma, \gamma}\right) g^{\rho \gamma^{\prime}}\left(\delta_{\alpha}^{t} \delta_{\gamma^{\prime}}^{\mu} \delta_{\beta}^{\nu}+\delta_{\beta}^{t} \delta_{\alpha}^{\mu} \delta_{\gamma^{\prime}}^{\nu}-\delta_{\gamma^{\prime}}^{t} \delta_{\alpha}^{\mu} \delta_{\beta}^{\nu}\right)\right]
$$

For convenience and simplicity and no losing generality, we transform eq.(2.11) as coveriant 3-order tensor

$$
\begin{gathered}
\pi_{\lambda \theta \chi}(\mathbf{x}, t)=g_{\tau \lambda} g_{\mu \theta} g_{\nu \chi} \pi^{\tau \mu \nu}(\mathbf{x}, t)= \\
\kappa\left[\frac{-1}{2} g_{\tau \lambda} g_{\mu \theta} g_{\nu \chi} g^{\alpha \beta} g^{\sigma \tau^{\prime}} \delta_{\beta}^{\tau} \delta_{\tau^{\prime}}^{\mu} \delta_{\varepsilon}^{\nu} g^{\varepsilon \gamma}\left(g_{\gamma \sigma, \alpha}+g_{\alpha \gamma, \sigma}-g_{\alpha \sigma, \gamma}\right)\right. \\
-\frac{1}{2} g_{\tau \lambda} g_{\mu \theta} g_{\nu \chi} g^{\alpha \beta} g^{\sigma \tau^{\prime}} g_{\tau^{\prime} \varepsilon, \beta} g^{\varepsilon \gamma}\left(\delta_{\alpha}^{\tau} \delta_{\gamma}^{\mu} \delta_{\sigma}^{\nu}+\delta_{\sigma}^{\tau} \delta_{\alpha}^{\mu} \delta_{\gamma}^{\nu}-\delta_{\gamma}^{\tau} \delta_{\alpha}^{\mu} \delta_{\sigma}^{\nu}\right) \\
+\frac{1}{2} g_{\tau \lambda} g_{\mu \theta} g_{\nu \chi} g^{\alpha \beta} g^{\sigma \tau^{\prime}} \delta_{\sigma}^{\tau} \delta_{\tau^{\prime}}^{\mu} \delta_{\varepsilon}^{\nu} g^{\varepsilon \gamma}\left(g_{\gamma \beta, \alpha}+g_{\alpha \gamma, \beta}-g_{\alpha \beta, \gamma}\right) \\
+\frac{1}{2} g_{\tau \lambda} g_{\mu \theta} g_{\nu \chi} g^{\alpha \beta} g^{\sigma \tau^{\prime}} g_{\tau^{\prime} \varepsilon, \sigma} g^{\varepsilon \gamma}\left(\delta_{\alpha}^{\tau} \delta_{\gamma}^{\mu} \delta_{\beta}^{\nu}+\delta_{\beta}^{\tau} \delta_{\alpha}^{\mu} \delta_{\gamma}^{\nu}-\delta_{\gamma}^{\tau} \delta_{\alpha}^{\mu} \delta_{\beta}^{\nu}\right) \mathrm{at}
\end{gathered}
$$$$
) g_{\tau \lambda} g_{\mu \theta} g_{\nu \chi} g^{\alpha \beta} g^{\sigma \gamma} g^{\rho \gamma^{\prime}}\left(\delta_{\alpha}^{\tau} \delta_{\gamma^{\prime}}^{\mu} \delta_{\beta}^{\nu}+\delta_{\beta}^{\tau} \delta_{\alpha}^{\mu} \delta_{\gamma^{\prime}}^{\nu}-\delta_{\gamma^{\prime}}^{\tau} \delta_{\alpha}^{\mu} \delta_{\beta}^{\nu}\right)\right]
$$

$$
\begin{aligned}
& +\frac{1}{4} g_{\tau \lambda} g_{\mu \theta} g_{\nu \chi} g^{\alpha \beta} g^{\sigma \gamma} g^{\rho \gamma^{\prime}}\left(\delta_{\rho}^{\tau} \delta_{\gamma}^{\mu} \delta_{\beta}^{\nu}+\delta_{\beta}^{\tau} \delta_{\rho}^{\mu} \delta_{\gamma}^{\nu}-\delta_{\gamma}^{\tau} \delta_{\rho}^{\mu} \delta_{\beta}^{\nu}\right)( \\
& \left.g_{\gamma^{\prime} \sigma, \alpha}+g_{\alpha \gamma^{\prime}, \sigma}-g_{\alpha \sigma, \gamma^{\prime}}\right)+\frac{1}{4}\left(g_{\gamma \beta, \rho}+g_{\rho \gamma, \beta}-g_{\rho \beta, \gamma}\right. \\
& ) g_{\tau \lambda} g_{\mu \theta} g_{\nu \chi} g^{\alpha \beta} g^{\sigma \gamma} g^{\rho \gamma^{\prime}}\left(\delta_{\alpha}^{\tau} \delta_{\gamma^{\prime}}^{\mu} \delta_{\sigma}^{\nu}+\delta_{\sigma}^{\tau} \delta_{\alpha}^{\mu} \delta_{\gamma^{\prime}}^{\nu}-\delta_{\gamma^{\prime}}^{\tau} \delta_{\alpha}^{\mu} \delta_{\sigma}^{\nu}\right) \\
& -\frac{1}{4} g_{\tau \lambda} g_{\mu \theta} g_{\nu \chi} g^{\alpha \beta} g^{\sigma \gamma} g^{\rho \gamma^{\prime}}\left(\delta_{\rho}^{\tau} \delta_{\gamma}^{\mu} \delta_{\sigma}^{\nu}+\delta_{\sigma}^{\tau} \delta_{\rho}^{\mu} \delta_{\gamma}^{\nu}-\delta_{\gamma}^{\tau} \delta_{\rho}^{\mu} \delta_{\sigma}^{\nu}\right. \\
& )\left(g_{\gamma^{\prime} \beta, \alpha}+g_{\alpha \gamma^{\prime}, \beta}-g_{\alpha \beta, \gamma^{\prime}}\right)-\frac{1}{4}\left(g_{\gamma \sigma, \rho}+g_{\rho \gamma, \sigma}-g_{\rho \sigma, \gamma}\right.
\end{aligned}
$$

Using eq.(2.12), we finally achieve

$$
\pi_{\lambda \theta \chi}(\mathbf{x}, t)=g_{\tau \lambda} g_{\mu \theta} g_{\nu \chi} \pi^{\tau \mu \nu}(\mathbf{x}, t)=\kappa\left[\frac{-3}{2} g_{\chi \theta, \lambda}+g_{\lambda \theta, \chi}+\frac{1}{2} g_{\lambda \chi} g_{\alpha \theta}^{, \alpha}+\right.
$$

$\left.\frac{3 g_{\lambda \theta} g_{\alpha \chi}^{, \alpha}}{2}-g_{\theta \chi} g_{\lambda \alpha}^{, \alpha}-\frac{g_{\lambda \chi} g^{\alpha \beta} g_{\alpha \beta, \theta}}{4}-\frac{3 g_{\lambda \theta} g^{\alpha \beta} g_{\alpha \beta, \chi}}{4}+\frac{g_{\theta \chi} g^{\alpha \beta} g_{\alpha \beta, \lambda}}{2}\right]$

where the detail calculations see appendix A in supplied net material.

\section{ENERGY OF GRAVITATIONAL FIELD OF THE STANDARD GRAVITATIONAL \\ LAGRANGIAN}

Using action (2.4) of gravitational field of the standard gravtational Lagrangian

$$
A=\int \mathcal{L} \sqrt{-g} d x^{4}=\int \kappa R \sqrt{-g} d x^{4}
$$

we get the energy of gravitational field of the standard gravtational Lagrangian

$$
\begin{gathered}
H=\int\left(\pi^{\mu \nu}(\mathbf{x}, t) g_{\mu \nu, t}(\mathbf{x}, t)-\mathcal{L}\right) \sqrt{-g} d x^{4}= \\
\int \kappa\left(\frac{g^{\alpha \beta} \partial R_{\alpha \beta}}{\partial \partial_{t} g_{\mu \nu}(\mathbf{x}, t)} g_{\mu \nu, t}(\mathbf{x}, t)-R\right) \sqrt{-g} d x^{4} .
\end{gathered}
$$

Eq.(3.2) means that if taking

$$
\left.\pi^{\mu \nu}(\mathbf{x}, t)\right]=\frac{\partial \mathcal{L}}{\partial \partial_{t} g_{\mu \nu}(\mathbf{x}, t)}=\kappa \sqrt{-g} \frac{\partial R}{\partial \partial_{t} g_{\mu \nu}(\mathbf{x}, t)},
$$

then the canonical momentum needs not to differentiate $\sqrt{-g}$ in eq.(3.3), consequently, taking eq.(2.5) as the 
canonical momentum is consistent and the most economic, or all momenta will be with $\sqrt{-g}$ througth the whole paper.

Using eq.(2.13), we deduce three order contravariant tensor

$$
\begin{gathered}
\pi^{\gamma \alpha^{\prime} \beta^{\prime}}(\mathbf{x}, t)=g^{\gamma \lambda} g^{\alpha^{\prime} \theta} g^{\beta^{\prime}} \pi_{\lambda \theta \chi}(\mathbf{x}, t)=g^{\gamma \lambda} g^{\alpha^{\prime} \theta} g^{\beta^{\prime}} \chi_{\tau \lambda} g_{\mu \theta} . \\
g_{\nu \chi} \pi^{\tau \mu \nu}(\mathbf{x}, t)=\pi^{\gamma \alpha^{\prime} \beta^{\prime}}(\mathbf{x}, t)=\kappa\left[\frac{-3}{2} g^{\gamma \lambda} g^{\alpha^{\prime} \theta} g^{\beta^{\prime}} g_{\chi \theta, \lambda}+\right. \\
g^{\gamma \lambda} g^{\alpha^{\prime} \theta} g^{\beta^{\prime} \chi} g_{\lambda \theta, \chi}+\frac{1}{2} g^{\gamma \lambda} g^{\alpha^{\prime} \theta} g^{\beta^{\prime} \chi} g_{\lambda \chi} g_{\alpha \theta}^{, \alpha}+\frac{3}{2} g^{\gamma \lambda} g^{\alpha^{\prime} \theta} g^{\beta^{\prime}} g_{\lambda \theta} g_{\alpha \chi}^{, \alpha} \\
-g^{\gamma \lambda} g^{\alpha^{\prime} \theta} g^{\beta^{\prime} \chi} g_{\theta \chi} g_{\lambda \alpha}^{, \alpha}-\frac{1}{4} g^{\gamma \lambda} g^{\alpha^{\prime} \theta} g^{\beta^{\prime} \chi} g_{\lambda \chi} g^{\alpha \beta} g_{\alpha \beta, \theta}
\end{gathered}
$$$$
\left.-\frac{3}{4} g^{\gamma \lambda} g^{\alpha^{\prime} \theta} g^{\beta^{\prime} \chi} g_{\lambda \theta} g^{\alpha \beta} g_{\alpha \beta, \chi}+\frac{1}{2} g^{\gamma \lambda} g^{\alpha^{\prime} \theta} g^{\beta^{\prime} \chi} g_{\theta \chi} g^{\alpha \beta} g_{\alpha \beta, \lambda}\right]=\kappa[-
$$$$
\frac{3}{2} g^{\beta^{\prime} \alpha^{\prime}, \gamma}+g^{\gamma \alpha^{\prime}, \beta^{\prime}}+\frac{1}{2} g^{\alpha^{\prime} \theta} g^{\gamma \beta^{\prime}} g_{\alpha \theta}^{, \alpha}+\frac{3}{2} g^{\beta^{\prime} \chi} g_{\lambda \theta}^{\gamma \alpha^{\prime}} g_{\alpha \chi}^{, \alpha}-g^{\gamma \lambda} g^{\alpha^{\prime} \beta^{\prime}} g_{\lambda \alpha}^{, \alpha}
$$$$
\left.-\frac{1}{4} g^{\gamma \beta^{\prime}} g^{\alpha \beta} g_{\alpha \beta}^{, \alpha^{\prime}}-\frac{3}{4} g^{\gamma \alpha^{\prime}} g^{\alpha \beta} g_{\alpha \beta}^{\beta^{\prime}}+\frac{1}{2} g^{\alpha^{\prime} \beta^{\prime}} g^{\alpha \beta} g_{\alpha \beta}^{, \gamma}\right]
$$

Because the index $\gamma$ is relevant to the time derivative, we take $\gamma$ as $t$ in eq.(3.4), then we get the two order contravariant tensor

$$
\begin{gathered}
\pi^{\mu \nu}(\mathbf{x}, t) \equiv \pi^{t \mu \nu}(\mathbf{x}, t)=\kappa\left(\frac{-3}{2} g^{\mu \nu, t}+g^{t \mu, \nu}\right. \\
+\frac{1}{2} g^{\mu \theta} g^{t \nu} g_{\alpha \theta}^{, \alpha}+\frac{3}{2} g^{\nu \chi} g^{t \mu} g_{\alpha \chi}^{, \alpha}-g^{t \lambda} g^{\mu \nu} g_{\lambda \alpha}^{, \alpha}- \\
\left.\frac{1}{4} g^{t \nu} g^{\alpha \beta} g_{\alpha \beta}^{, \mu}-\frac{3}{4} g^{t \mu} g^{\alpha \beta} g_{\alpha \beta}^{, \nu}+\frac{1}{2} g^{\mu \nu} g^{\alpha \beta} g_{\alpha \beta}^{, t}\right) .
\end{gathered}
$$

Substituting eq.(3.5) into eq.(3.2), we deduce

$$
\begin{gathered}
H=\int\left(\pi^{\mu \nu}(\mathbf{x}, t) g_{\mu \nu, t}(\mathbf{x}, t)-\mathcal{L}\right) \sqrt{-g} d x^{4}= \\
H=\int \kappa\left[\left(\frac{-3}{2} g^{\mu \nu, t}+g^{t \mu, \nu}+\frac{1}{2} g^{\mu \theta} g^{t \nu} g_{\alpha \theta}^{, \alpha}+\right.\right. \\
\frac{3}{2} g^{\nu \chi} g^{t \mu} g_{\alpha \chi}^{, \alpha}-g^{t \lambda} g^{\mu \nu} g_{\lambda \alpha}^{, \alpha}-\frac{1}{4} g^{t \nu} g^{\alpha \beta} g_{\alpha \beta}^{, \mu}-\frac{3}{4} g^{t \mu} g^{\alpha \beta} g_{\alpha \beta}^{, \nu} \\
\left.\left.\left.+\frac{1}{2} g^{\mu \nu} g^{\alpha \beta} g_{\alpha \beta}^{, t}\right)\right) g_{\mu \nu, t}(\mathbf{x}, t)-g^{\alpha \beta} R_{\alpha \beta}\right] \sqrt{-g} d x^{4} .
\end{gathered}
$$

It is very easy to calculate the standard gravitational system energy eq.(3.6) when substituting concrete metric models into eq.(3.6).

\section{FURTHER INVESTIGATIONS OF QUANTIZATION OF QUANTUM GRAVITATIONAL FIELDS}

Because commutation relations of different fields and their canonical momenta play very key role in quantization theory, we now study the commutation relations.

For convenience and no losing generality, using eq.(3.5), we can define

$$
\begin{gathered}
\pi^{\mu \nu}(\mathbf{x}, t) \equiv \pi^{(0) \mu \nu}(\mathbf{x}, t)+\pi^{\prime(1) \mu \nu}(\mathbf{x}, t)+\pi^{\prime(2) \mu \nu}(\mathbf{x}, t) \\
+\pi^{\prime(3) \mu \nu}(\mathbf{x}, t)=\pi^{(3) \mu \nu}(\mathbf{x}, t)=\pi^{\prime(3) \mu \nu}(\mathbf{x}, t)+ \\
\pi^{(2) \mu \nu}(\mathbf{x}, t)=\pi^{\prime(3) \mu \nu}(\mathbf{x}, t)+\pi^{\prime(2) \mu \nu}(\mathbf{x}, t)+\pi^{(1) \mu \nu}(\mathbf{x}, t),
\end{gathered}
$$

$$
\pi^{(0) \mu \nu}(\mathbf{x}, t)=\kappa \frac{-3}{2} g^{\mu \nu, t},
$$

$$
\pi^{\prime(1) \mu \nu}(\mathbf{x}, t)=\kappa g^{t \mu, \nu}
$$

$\pi^{\prime(2) \mu \nu}(\mathbf{x}, t)=\kappa\left(\frac{1}{2} g^{\mu \theta} g^{t \nu} g_{\alpha \theta}^{, \alpha}+\frac{3}{2} g^{\nu \chi} g^{t \mu} g_{\alpha \chi}^{, \alpha}-g^{t \lambda} g^{\mu \nu} g_{\lambda \alpha}^{, \alpha}\right)$

$\pi^{\prime(3) \mu \nu}(\mathbf{x}, t)=\kappa\left(\frac{1}{2} g^{\mu \nu} g^{\alpha \beta} g_{\alpha \beta}^{, t}-\frac{1}{4} g^{t \nu} g^{\alpha \beta} g_{\alpha \beta}^{, \mu}-\frac{3}{4} g^{t \mu} g^{\alpha \beta} g_{\alpha \beta}^{, \nu}\right)$

For $\left[\hat{g}_{\mu \nu}(\mathbf{x}, t), \hat{\pi}^{(0) \alpha \beta}\left(\mathbf{x}^{\prime}, t^{\prime}\right)\right]_{t=t^{\prime}}$, we may define

$$
\hat{\pi}^{(0) \mu \nu}(\mathbf{x}, t)=-i \frac{\partial}{\partial \hat{g}_{\mu \nu}(\mathbf{x}, t)},
$$

then the classical Poisson bracket for any operators needs to be taken as

$$
\begin{gathered}
\left\{\hat{X}(\mathbf{x}, t), \hat{Y}\left(\mathbf{x}^{\prime}, t^{\prime}\right)\right\}_{p b, t=t^{\prime}}= \\
\int\left(\frac{\partial \hat{X}(x)}{\partial \hat{g}_{\mu \nu}(y)} \frac{\partial \hat{Y}\left(x^{\prime}\right)}{\partial \hat{\pi}^{(0) \mu \nu}(y)}-\frac{\partial \hat{X}(x)}{\partial \hat{\pi}^{(0) \mu \nu}(y)} \frac{\partial \hat{Y}\left(x^{\prime}\right)}{\partial \hat{g}_{\mu \nu}(y)}\right)_{p b, t=t^{\prime}} d \mathbf{y}
\end{gathered}
$$

so that we can obtain the consistent theory between operator commutation relations and classical Poisson brackets.

Therefore, we naturally have commutation relation of field operator and its canonical conjugate momentum in the quantization for infinite degrees of freedom

$\frac{1}{i}\left[\hat{g}_{\mu \nu}(\mathbf{x}, t), \hat{\pi}^{(0) \alpha \beta}\left(\mathbf{x}^{\prime}, t^{\prime}\right)\right]_{t=t^{\prime}}=\frac{1}{i}\left[\hat{g}_{\mu \nu}(\mathbf{x}, t),-i \frac{\partial}{\partial \hat{g}_{\alpha \beta}\left(\mathbf{x}^{\prime}, t\right)}\right]_{t=t^{\prime}}$ 


$$
\begin{gathered}
=\delta_{\mu}^{\alpha} \delta_{\nu}^{\beta} \delta\left(\mathbf{x}-\mathbf{x}^{\prime}\right)=\Delta_{\mu \nu}^{(0) \alpha \beta} \delta\left(\mathbf{x}-\mathbf{x}^{\prime}\right)=-\hat{g}_{\mu \nu}(\mathbf{x}, t) \frac{\partial}{\partial \hat{g}_{\alpha \beta}(\mathbf{x}, t)}+ \\
\hat{g}_{\mu \nu}(\mathbf{x}, t) \frac{\partial}{\partial \hat{g}_{\alpha \beta}(\mathbf{x}, t)}+\frac{\partial \hat{g}_{\mu \nu}(\mathbf{x}, t)}{\partial \hat{g}_{\alpha \beta}(\mathbf{x}, t)}=\int( \\
\frac{\partial \hat{g}_{\mu \nu}(\mathbf{x}, t)}{\partial \hat{g}_{\rho \sigma}(y)} \frac{\partial \hat{\pi}^{(0) \alpha \beta}\left(\mathbf{x}^{\prime}, t^{\prime}\right)}{\partial \hat{\pi}^{(0) \rho \sigma}(y)}-\frac{\partial \hat{g}_{\mu \nu}(\mathbf{x}, t)}{\partial \hat{\pi}^{(0) \rho \sigma}(y)} \frac{\partial \hat{\pi}^{(0) \alpha \beta}\left(\mathbf{x}^{\prime}, t^{\prime}\right)}{\partial \hat{g}_{\rho \sigma}(y)} \\
)_{p b, t=t^{\prime}=t_{y}} d \mathbf{y}=\left\{\hat{g}_{\mu \nu}(\mathbf{x}, t), \hat{\pi}^{(0) \alpha \beta}\left(\mathbf{x}^{\prime}, t^{\prime}\right)\right\}_{p b, t=t^{\prime}},
\end{gathered}
$$

where $\left\{\hat{g}_{\mu \nu}(\mathbf{x}, t), \hat{\pi}^{(0) \alpha \beta}\left(\mathbf{x}^{\prime}, t^{\prime}\right)\right\}_{p b, t=t^{\prime}}$ is classical Poisson bracket for infinite degrees of freedom.

For infinite degrees of freedom and similar to investigations on Eq.(4.8), we have

$$
\begin{array}{cc}
\frac{1}{i}\left[\hat{g}_{\mu \nu}(\mathbf{x}, t), \hat{g}_{\alpha \beta}\left(\mathbf{x}^{\prime}, t\right)\right]_{t=t^{\prime}}=\frac{1}{i}\left[\hat{g}_{\mu \nu}(\mathbf{x}, t) \hat{g}_{\alpha \beta}\left(\mathbf{x}^{\prime}, t\right)-\right. & \text { Thus } \\
\left.\hat{g}_{\alpha \beta}\left(\mathbf{x}^{\prime}, t\right) \hat{g}_{\mu \nu}(\mathbf{x}, t)\right]_{t=t^{\prime}}=0=\int\left(\frac{\partial \hat{g}_{\mu \nu}(\mathbf{x}, t)}{\partial \hat{g}_{\rho \sigma}(y)} \frac{\partial \hat{g}_{\alpha \beta}\left(\mathbf{x}^{\prime}, t^{\prime}\right)}{\partial \hat{\pi}^{(0) \rho \sigma}(y)}-\right. & \quad(4.9) \\
\left.\frac{\partial \hat{g}_{\mu \nu}(\mathbf{x}, t)}{\partial \hat{\pi}^{(0) \rho \sigma}(y)} \frac{\partial \hat{g}_{\alpha \beta}\left(\mathbf{x}^{\prime}, t^{\prime}\right)}{\partial \hat{g}_{\rho \sigma}(y)}\right)_{p b, t=t^{\prime}=t_{y}} d \mathbf{y}=\left\{\hat{g}_{\mu \nu}(\mathbf{x}, t), \hat{g}_{\alpha \beta}\left(\mathbf{x}^{\prime}, t^{\prime}\right)\right\}_{p b, t=t^{\prime}} \\
\frac{1}{i}\left[\hat{\pi}^{(0) \mu \nu}(\mathbf{x}, t), \hat{\pi}^{(0) \alpha \beta}\left(\mathbf{x}^{\prime}, t^{\prime}\right)\right]_{t=t^{\prime}}=\frac{1}{i}\left[\hat{\pi}^{(0) \mu \nu}(\mathbf{x}, t) \hat{\pi}^{(0) \alpha \beta}\left(\mathbf{x}^{\prime}, t^{\prime}\right)\right. \\
\left.-\hat{\pi}^{(0) \alpha \beta}\left(\mathbf{x}^{\prime}, t^{\prime}\right) \hat{\pi}^{(0) \mu \nu}(\mathbf{x}, t)\right]_{t=t^{\prime}}=0=\int( & \text { where } \\
\frac{\partial \hat{\pi}^{(0) \mu \nu}(\mathbf{x}, t)}{\partial \hat{g}_{\rho \sigma}(y)} \frac{\partial \hat{\pi}^{(0) \alpha \beta}\left(\mathbf{x}^{\prime}, t^{\prime}\right)}{\partial \hat{\pi}^{(0) \rho \sigma}(y)}-\frac{\partial \hat{\pi}^{(0) \mu \nu}(\mathbf{x}, t)}{\partial \hat{\pi}^{(0) \rho \sigma}(y)} \frac{\partial \hat{\pi}^{(0) \alpha \beta}\left(\mathbf{x}^{\prime}, t^{\prime}\right)}{\partial \hat{g}_{\rho \sigma}(y)} & \begin{array}{l}
\text { property } \\
\text { nonlin } \\
\text { order }
\end{array} \\
)_{p b, t=t^{\prime}=t} d \mathbf{y}=\left\{\hat{\pi}^{(0) \mu \nu}(\mathbf{x}, t), \hat{\pi}^{(0) \alpha \beta}\left(\mathbf{x}^{\prime}, t^{\prime}\right)\right\}_{p b, t=t^{\prime}} &
\end{array}
$$

Using the investigations of this section, we can give many important investigations. In terms of the detailed argument in this section, we do find that, for the commutative relations of operators, people can do exact calculations directly with the classical Poisson's bracket of operators because they are completely equivalent by the relations above.

\section{COMMUTATION RELATION FOR GRAVITATIONAL FIELDS AND THE FIRST STYLE OF MOMENTA}

We further consider the commutation relation for gravitational fields and the first style of momenta $\hat{\pi}^{(1) \alpha \beta}\left(\mathbf{x}^{\prime}, t^{\prime}\right)$

$$
\frac{1}{i}\left[\hat{g}_{\mu \nu}(\mathbf{x}, t), \hat{\pi}^{(1) \alpha \beta}\left(\mathbf{x}^{\prime}, t^{\prime}\right)\right]_{t=t^{\prime}}=\frac{1}{i}\left[\hat{g}_{\mu \nu}(\mathbf{x}, t), \hat{\pi}^{(0) \alpha \beta}\left(\mathbf{x}^{\prime}, t^{\prime}\right)\right]_{t=t^{\prime}}
$$$$
+\frac{1}{i}\left[\hat{g}_{\mu \nu}(\mathbf{x}, t), \hat{\pi}^{\prime(1) \alpha \beta}\left(\mathbf{x}^{\prime}, t^{\prime}\right)\right]_{t=t^{\prime}}=\frac{1}{i}\left[\hat{g}_{\mu \nu}(\mathbf{x}, t), \hat{\pi}^{(0) \alpha \beta}\left(\mathbf{x}^{\prime}, t^{\prime}\right)\right.
$$$$
]_{t=t^{\prime}}+\frac{1}{i}\left[\hat{g}_{\mu \nu}(\mathbf{x}, t), \kappa g^{t \alpha, \beta}\right]_{t=t^{\prime}}=\frac{1}{i}\left[\hat{g}_{\mu \nu}(\mathbf{x}, t), \hat{\pi}^{(0) \alpha \beta}\left(\mathbf{x}^{\prime}, t^{\prime}\right)\right]_{t=t^{\prime}}+
$$$$
\int\left(\frac{\partial \hat{g}_{\mu \nu}(\mathbf{x}, t)}{\partial \hat{g}_{\rho \sigma}(y)} \frac{\partial\left(\kappa g^{t \alpha, \beta}\right)}{\partial\left(\kappa \frac{-3}{2} g^{\rho \sigma, t}\right)}-\frac{\partial \hat{g}_{\mu \nu}(\mathbf{x}, t)}{\partial \hat{\pi}^{(0) \rho \sigma}(y)} \frac{\partial \hat{\pi}^{\prime(1) \alpha \beta}\left(\mathbf{x}^{\prime}, t^{\prime}\right)}{\partial \hat{g}_{\rho \sigma}(y)}\right.
$$

$$
)_{p b, t=t^{\prime}=t_{y}} d \mathbf{y}=\left\{\hat{g}_{\mu \nu}(\mathbf{x}, t), \hat{\pi}^{(1) \alpha \beta}\left(\mathbf{x}^{\prime}, t^{\prime}\right)\right\}_{p b, t=t^{\prime}} .
$$

Thus we can further deduce

$$
\begin{aligned}
& \frac{1}{i}\left[\hat{g}_{\mu \nu}(\mathbf{x}, t), \hat{\pi}^{(1) \alpha \beta}\left(\mathbf{x}^{\prime}, t^{\prime}\right)\right]_{t=t^{\prime}}=\delta_{\mu}^{\alpha} \delta_{\nu}^{\beta} \delta\left(\mathbf{x}-\mathbf{x}^{\prime}\right) \\
& -\frac{2}{3} \int\left(\delta_{\mu}^{\rho} \delta_{\nu}^{\sigma} \delta(\mathbf{x}-\mathbf{y}) \delta_{\rho}^{t} \delta_{\sigma}^{\alpha} \delta_{t}^{\beta} \delta\left(\mathbf{x}^{\prime}-\mathbf{y}\right)\right)_{p b, t=t^{\prime}=t_{y}} d \mathbf{y}
\end{aligned}
$$

$$
\begin{gathered}
=\delta_{\mu}^{\alpha} \delta_{\nu}^{\beta} \delta\left(\mathbf{x}-\mathbf{x}^{\prime}\right)-\frac{2}{3} \delta_{\mu}^{t} \delta_{\nu}^{\alpha} \delta\left(\mathbf{x}-\mathbf{x}^{\prime}\right) \delta_{t}^{\beta} \\
=\left(\delta_{\mu}^{\alpha} \delta_{\nu}^{\beta}-\frac{2}{3} \delta_{\mu}^{\beta} \delta_{\nu}^{\alpha}\right) \delta\left(\mathbf{x}-\mathbf{x}^{\prime}\right)=\Delta_{\mu \nu}^{(1) \alpha \beta} \delta\left(\mathbf{x}-\mathbf{x}^{\prime}\right),
\end{gathered}
$$

where the first term and the second term on right side of eq.(5.2) are linear terms, which shows the only linear property of the standard gravitational Lagrangian, the nonlinear property will be shown in the following higher order term studies.

\section{COMMUTATION RELATION FOR GRAVITATIONAL FIELDS AND THE SECOND STYLE OF MOMENTA}

We now consider the commutation relation for gravitational fields and the second style of momenta $\hat{\pi}^{(2) \alpha \beta}\left(\mathbf{x}^{\prime}, t^{\prime}\right)$

$$
\begin{aligned}
& \frac{1}{i}\left[\hat{g}_{\mu^{\prime} \nu^{\prime}}(\mathbf{x}, t), \hat{\pi}^{(2) \mu \nu}\left(\mathbf{x}^{\prime}, t^{\prime}\right)\right]_{t=t^{\prime}}=\frac{1}{i}\left[\hat{g}_{\mu^{\prime} \nu^{\prime}}(\mathbf{x}, t), \hat{\pi}^{(1) \mu \nu}\left(\mathbf{x}^{\prime}, t^{\prime}\right)\right. \\
& ]_{t=t^{\prime}}+\frac{1}{i}\left[\hat{g}_{\mu^{\prime} \nu^{\prime}}(\mathbf{x}, t), \hat{\pi}^{\prime(2) \mu \nu}\left(\mathbf{x}^{\prime}, t^{\prime}\right)\right]_{t=t^{\prime}}=\frac{1}{i}\left[\hat{g}_{\mu^{\prime} \nu^{\prime}}(\mathbf{x}, t), \hat{\pi}^{(1) \mu \nu}(\right.
\end{aligned}
$$

$\left.\left.\mathbf{x}^{\prime}, t^{\prime}\right)\right]_{t=t^{\prime}}+\frac{1}{i}\left[\hat{g}_{\mu^{\prime} \nu^{\prime}}(\mathbf{x}, t), \kappa\left(\frac{1}{2} g^{\mu \theta} g^{t \nu} g_{\alpha \theta}^{, \alpha}+\frac{3}{2} g^{\nu \chi} g^{t \mu} g_{\alpha \chi}^{, \alpha}-g^{t \lambda} g^{\mu \nu} g_{\lambda \alpha}^{, \alpha}\right.\right.$ 
Preprints (www.preprints.org)

NOT PEER-REVIEWED

Posted: 8 July 2021

doi:10.20944/preprints202107.0054.v2

6

$$
\begin{aligned}
& )]_{t=t^{\prime}}=\frac{1}{i}\left[\hat{g}_{\mu^{\prime} \nu^{\prime}}(\mathbf{x}, t), \hat{\pi}^{(1) \mu \nu}\left(\mathbf{x}^{\prime}, t^{\prime}\right)\right]_{t=t^{\prime}}+\int\left(\frac{\partial \hat{g}_{\mu^{\prime} \nu^{\prime}}(\mathbf{x}, t)}{\partial \hat{g}_{\rho \sigma}(y)} . \quad g^{\mu \nu} \delta_{\rho}^{t} \delta_{\sigma^{\prime}}^{\sigma} \delta_{t}^{\alpha} g_{\sigma \alpha}\right) \delta\left(\mathbf{y}-\mathbf{x}^{\prime}\right)=\frac{1}{i}\left[\hat{g}_{\mu^{\prime} \nu^{\prime}}(\mathbf{x}, t), \hat{\pi}^{(1) \mu \nu}\left(\mathbf{x}^{\prime}, t^{\prime}\right)\right]_{t=t^{\prime}}+ \\
& \frac{\partial \kappa\left(\frac{1}{2} g^{\mu \theta} g^{t \nu} g^{\alpha \gamma} g_{\alpha \theta, \gamma}+\frac{3}{2} g^{\nu \chi} g^{t \mu} g^{\alpha \gamma} g_{\alpha \chi, \gamma}-g^{t \lambda} g^{\mu \nu} g^{\alpha \gamma} g_{\lambda \alpha, \gamma}\right)}{\partial\left(\kappa \frac{-3}{2} g^{\rho \sigma, t}\right)} \quad\left(\delta _ { \mu ^ { \prime } } ^ { \rho } \delta _ { \nu ^ { \prime } } ^ { \sigma ^ { \prime } } \kappa \left(\frac{1}{2} g^{t \nu}\left(-g_{\alpha \sigma} \delta_{\rho}^{\sigma} \delta_{\sigma^{\prime}}^{\mu} \delta_{t}^{\alpha}\right)+\frac{3}{2} g^{t \mu}\left(-g_{\alpha \sigma} \delta_{\rho}^{\sigma} \delta_{\sigma^{\prime}}^{\nu} \delta_{t}^{\alpha}\right)-\right.\right. \\
& )_{p b, t=t^{\prime}=t_{y}} d \mathbf{y}=\left\{\hat{g}_{\mu^{\prime} \nu^{\prime}}(\mathbf{x}, t), \hat{\pi}^{(2) \mu \nu}\left(\mathbf{x}^{\prime}, t^{\prime}\right)\right\}_{p b, t=t^{\prime}}=\frac{1}{i}[ \\
& \left.g^{\mu \nu}\left(-\delta_{\rho}^{t} \delta_{\sigma^{\prime}}^{\sigma} \delta_{t}^{\alpha} g_{\sigma \alpha}\right)\right) \delta\left(\mathbf{x}-\mathbf{x}^{\prime}\right)=\frac{1}{i}[ \\
& \left.\hat{g}_{\mu^{\prime} \nu^{\prime}}(\mathbf{x}, t), \hat{\pi}^{(1) \mu \nu}\left(\mathbf{x}^{\prime}, t^{\prime}\right)\right]_{t=t^{\prime}}+\int d \mathbf{y}\left(\frac{\partial \hat{g}_{\mu^{\prime} \nu^{\prime}}(\mathbf{x}, t)}{\partial \hat{g}_{\rho \sigma}(y)} \frac{\partial}{\partial\left(\kappa \frac{-3}{2} g^{\rho \sigma, t}\right)} \kappa\left(\hat{g}_{\mu^{\prime} \nu^{\prime}}(\mathbf{x}, t), \hat{\pi}^{(1) \mu \nu}\left(\mathbf{x}^{\prime}, t^{\prime}\right)\right]_{t=t^{\prime}}+\left(\left(\frac{1}{2} g^{t \nu}\left(-g_{t \mu^{\prime}} \delta_{\nu^{\prime}}^{\mu}\right)+\frac{3}{2} g^{t \mu}\left(-g_{t \mu^{\prime}} \delta_{\nu^{\prime}}^{\nu}\right)\right.\right.\right. \\
& \left.\left.\left.\frac{1}{2} g^{\mu \theta} g^{t \nu} g^{\alpha \gamma} g_{\alpha \theta, \gamma}+\frac{3}{2} g^{\nu \chi} g^{t \mu} g^{\alpha \gamma} g_{\alpha \chi, \gamma}-g^{t \lambda} g^{\mu \nu} g^{\alpha \gamma} g_{\lambda \alpha, \gamma}\right)\right)_{t=t^{\prime}=t_{y}} \cdot-g^{\mu \nu}\left(-\delta_{\mu^{\prime}}^{t} g_{\nu^{\prime} t}\right)\right) \delta\left(\mathbf{x}-\mathbf{x}^{\prime}\right)=\frac{1}{i}\left[\hat{g}_{\mu^{\prime} \nu^{\prime}}(\mathbf{x}, t), \hat{\pi}^{(1) \mu \nu}\left(\mathbf{x}^{\prime}, t^{\prime}\right)\right]_{t=t^{\prime}} \\
& +\left(\left(-\frac{1}{2} \delta_{\mu^{\prime}}^{\nu} \delta_{\nu^{\prime}}^{\mu}\right)-\frac{3}{2} \delta_{\mu^{\prime}}^{\mu} \delta_{\nu^{\prime}}^{\nu}-g^{\mu \nu} g_{\nu^{\prime} \mu^{\prime}}\right) \delta\left(\mathbf{x}-\mathbf{x}^{\prime}\right) .
\end{aligned}
$$

$g^{\sigma \mu} g_{\mu \beta, \rho}=-g_{, \rho}^{\sigma \mu} g_{\mu \beta}, g_{\alpha \sigma} g^{\sigma \mu} g_{\mu \beta, \rho}=-g_{\alpha \sigma} g_{, \rho}^{\sigma \mu} g_{\mu \beta}=g_{\alpha \beta, \rho}$,

Putting eq.(5.2) into eq.(6.4), it follows that

we can have

$$
\begin{aligned}
& \hat{\pi}^{\prime(2) \mu \nu}\left(\mathbf{x}^{\prime}, t^{\prime}\right)=\kappa\left(\frac{1}{2} g^{\mu \theta} g^{t \nu} g^{\alpha \gamma} g_{\alpha \theta, \gamma}+\frac{3}{2} g^{\nu \chi} g^{t \mu} g^{\alpha \gamma} g_{\alpha \chi, \gamma}-\quad \frac{1}{i}\left[\hat{g}_{\mu^{\prime} \nu^{\prime}}(\mathbf{x}, t), \hat{\pi}^{(2) \mu \nu}\left(\mathbf{x}^{\prime}, t^{\prime}\right)\right]_{t=t^{\prime}}=\left(\delta_{\mu^{\prime}}^{\mu} \delta_{\nu^{\prime}}^{\nu}-\frac{2}{3} \delta_{\mu^{\prime}}^{\nu} \delta_{\nu^{\prime}}^{\mu}\right) \delta\left(\mathbf{x}-\mathbf{x}^{\prime}\right)\right. \\
& \left.g^{t \lambda} g^{\mu \nu} g^{\alpha \gamma} g_{\lambda \alpha, \gamma}\right)=\kappa\left(\frac{1}{2} g^{\mu \theta} g^{t \nu} g^{\alpha \gamma}\left(-g_{\alpha \sigma} g_{, \gamma}^{\sigma \mu^{\prime}} g_{\mu^{\prime} \theta}\right)+\frac{3}{2} g^{\nu \chi} g^{t \mu} g^{\alpha \gamma}\left(-\left(\left(-\frac{1}{2} \delta_{\mu^{\prime}}^{\nu} \delta_{\nu^{\prime}}^{\mu}\right)-\frac{3}{2} \delta_{\mu^{\prime}}^{\mu} \delta_{\nu^{\prime}}^{\nu}-g^{\mu \nu} g_{\nu^{\prime} \mu^{\prime}}\right) \delta\left(\mathbf{x}-\mathbf{x}^{\prime}\right)=-(\right.\right. \\
& \left.\left.g_{\alpha \sigma} g_{, \gamma}^{\sigma \mu^{\prime}} g_{\mu^{\prime} \chi}\right)-g^{t \lambda} g^{\mu \nu} g^{\alpha \gamma}\left(-g_{\lambda \mu^{\prime}} g_{, \gamma}^{\mu^{\prime} \sigma} g_{\sigma \alpha}\right)\right)=\kappa\left(\frac{1}{2} g^{\mu \theta} g^{t \nu}\left(-\quad \frac{1}{2} \delta_{\mu^{\prime}}^{\nu} \delta_{\nu^{\prime}}^{\mu}+\frac{7}{6} \delta_{\mu^{\prime}}^{\mu} \delta_{\nu^{\prime}}^{\nu}+g^{\mu \nu} g_{\nu^{\prime} \mu^{\prime}}\right) \delta\left(\mathbf{x}-\mathbf{x}^{\prime}\right)=\Delta_{\mu^{\prime} \nu^{\prime}}^{(2) \mu \nu} \delta\left(\mathbf{x}-\mathbf{x}^{\prime}\right),\right.
\end{aligned}
$$

$$
\left.g_{\alpha \sigma} g^{\sigma \mu^{\prime}, \alpha} g_{\mu^{\prime} \theta}\right)+\frac{3}{2} g^{\nu \chi} g^{t \mu}\left(-g_{\alpha \sigma} g^{\sigma \mu^{\prime}, \alpha} g_{\mu^{\prime} \chi}\right)-g^{t \lambda} g^{\mu \nu}(-
$$

where the first term and the second term on right side of eq.(6.5) are linear terms, the third term is nonlinear ter$\mathrm{m}$, which shows the nonlinear property of the nonlinear system of the standard gravitational Lagrangian.

$\left.\left.g_{\lambda \mu^{\prime}} g^{\mu^{\prime} \sigma, \alpha} g_{\sigma \alpha}\right)\right)=\kappa\left(\frac{1}{2} g^{t \nu}\left(-g_{\alpha \sigma} g^{\sigma \mu, \alpha}\right)+\frac{3}{2} g^{t \mu}\left(-g_{\alpha \sigma} g^{\sigma \nu, \alpha}\right)-g^{\mu \nu}\left(-g^{t \sigma, \alpha} g_{\sigma \alpha}\right)\right)$.

Putting eq.(6.3) into eq.(6.1), we have

VII. COMMUTATION RELATION FOR GRAVIATIONAL FIELDS AND THE THIRD STYLE OF MOMENTA

$\frac{1}{i}\left[\hat{g}_{\mu^{\prime} \nu^{\prime}}(\mathbf{x}, t), \hat{\pi}^{(2) \mu \nu}\left(\mathbf{x}^{\prime}, t^{\prime}\right)\right]_{t=t^{\prime}}=\frac{1}{i}\left[\hat{g}_{\mu^{\prime} \nu^{\prime}}(\mathbf{x}, t), \hat{\pi}^{(1) \mu \nu}\left(\mathbf{x}^{\prime}, t^{\prime}\right)\right]_{t=t^{\prime}}$ We now consider the commutation relation for gravitational fields and the third style of momenta $\hat{\pi}^{(3) \alpha \beta}\left(\mathbf{x}^{\prime}, t^{\prime}\right)$

$$
\begin{array}{cc}
+\kappa \int d \mathbf{y}\left(\frac { \partial \hat { g } _ { \mu ^ { \prime } \nu ^ { \prime } } ( \mathbf { x } , t ) } { \partial \hat { g } _ { \rho \sigma ^ { \prime } } ( y ) } \frac { \partial } { \partial ( \kappa \frac { - 3 } { 2 } g ^ { \rho \sigma ^ { \prime } , t } ) } \left(\frac{1}{2} g^{t \nu}\left(-g_{\alpha \sigma} g^{\sigma \mu, \alpha}\right)\right.\right. & \frac{1}{i}\left[\hat{g}_{\mu^{\prime} \nu^{\prime}}(\mathbf{x}, t), \hat{\pi}^{(3) \mu \nu}\left(\mathbf{x}^{\prime}, t^{\prime}\right)\right]_{t=t^{\prime}}=\frac{1}{i}\left[\hat{g}_{\mu^{\prime} \nu^{\prime}}(\mathbf{x}, t), \hat{\pi}^{(2) \mu \nu}\left(\mathbf{x}^{\prime}, t^{\prime}\right)\right. \\
\left.+\frac{3}{2} g^{t \mu}\left(-g_{\alpha \sigma} g^{\sigma \nu, \alpha}\right)-g^{\mu \nu}\left(-g^{t \sigma, \alpha} g_{\sigma \alpha}\right)\right)_{p b, t=t^{\prime}=t_{y}} & ]_{t=t^{\prime}}+\frac{1}{i}\left[\hat{g}_{\mu^{\prime} \nu^{\prime}}(\mathbf{x}, t), \hat{\pi}^{\prime(3) \mu \nu}\left(\mathbf{x}^{\prime}, t^{\prime}\right)\right]_{t=t^{\prime}}=\frac{1}{i}\left[\hat{g}_{\mu^{\prime} \nu^{\prime}}(\mathbf{x}, t), \hat{\pi}^{(2) \mu \nu}(\right. \\
=\frac{1}{i}\left[\hat{g}_{\mu^{\prime} \nu^{\prime}}(\mathbf{x}, t), \hat{\pi}^{(1) \mu \nu}\left(\mathbf{x}^{\prime}, t^{\prime}\right)\right]_{t=t^{\prime}}+\int d \mathbf{y}\left(\delta_{\mu^{\prime}}^{\rho} \delta_{\nu^{\prime}}^{\sigma^{\prime}} \delta(\mathbf{x}-\mathbf{y})(-\right. & \left.\left.\mathbf{x}^{\prime}, t^{\prime}\right)\right]_{t=t^{\prime}}+\frac{1}{i}\left[\hat{g}_{\mu^{\prime} \nu^{\prime}}(\mathbf{x}, t), \kappa\left(\frac{1}{2} g^{\mu \nu} g^{\alpha \beta} g_{\alpha \beta}^{t}-\frac{1}{4} g^{t \nu} g^{\alpha \beta} g_{\alpha \beta}^{, \mu}\right.\right. \\
\frac{g^{t \nu} g_{\alpha \sigma} \delta_{\rho}^{\sigma} \delta_{\sigma^{\prime}}^{\mu} \delta_{t}^{\alpha}}{2}+\frac{3 g^{t \mu}\left(-g_{\alpha \sigma} \delta_{\rho}^{\sigma} \delta_{\sigma^{\prime}}^{\nu} \delta_{t}^{\alpha}\right)}{2}+ & \left.\left.-\frac{3}{4} g^{t \mu} g^{\alpha \beta} g_{\alpha \beta}^{\nu}\right)\right]_{t=t^{\prime}}=\frac{1}{i}\left[\hat{g}_{\mu^{\prime} \nu^{\prime}}(\mathbf{x}, t), \hat{\pi}^{(2) \mu \nu}\left(\mathbf{x}^{\prime}, t^{\prime}\right)\right]_{t=t^{\prime}}+
\end{array}
$$




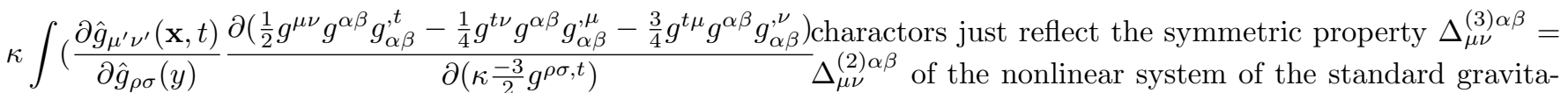
tional Lagrangian.

$$
)_{p b, t=t^{\prime}=t_{y}} d \mathbf{y}=\left\{\hat{g}_{\mu^{\prime} \nu^{\prime}}(\mathbf{x}, t), \hat{\pi}^{(3) \mu \nu}\left(\mathbf{x}^{\prime}, t^{\prime}\right)\right\}_{p b, t=t^{\prime}}
$$

Using eq.(4.5), we have

$$
\begin{aligned}
& \pi^{\prime(3) \mu \nu}(\mathbf{x}, t)=\kappa\left(\frac{1}{2} g^{\mu \nu} g^{\alpha \beta} g_{\alpha \beta}^{, t}-\frac{1}{4} g^{t \nu} g^{\alpha \beta} g_{\alpha \beta}^{, \mu}-\frac{3}{4} g^{t \mu} g^{\alpha \beta} g_{\alpha \beta}^{, \nu}\right) . \\
& =\kappa\left(\frac{1}{2} g^{\mu \nu} g^{\alpha \beta} g^{t \gamma}\left(-g_{\alpha \sigma} g_{, \gamma}^{\sigma \varepsilon} g_{\varepsilon \beta}\right)-\frac{1}{4} g^{t \nu} g^{\alpha \beta} g^{\mu \gamma}\left(-g_{\alpha \sigma} g_{, \gamma}^{\sigma \varepsilon} g_{\varepsilon \beta}\right)\right. \\
& \left.\quad-\frac{3}{4} g^{t \mu} g^{\alpha \beta} g^{\nu \gamma}\left(-g_{\alpha \sigma} g_{, \gamma}^{\sigma \varepsilon} g_{\varepsilon \beta}\right)\right) \\
& =\kappa\left(\frac{1}{2} g^{\mu \nu}\left(-g^{\beta \varepsilon, t} g_{\varepsilon \beta}\right)-\frac{1}{4} g^{t \nu}\left(-g^{\beta \varepsilon, \mu} g_{\varepsilon \beta}\right)-\frac{3}{4} g^{t \mu}\left(-g^{\beta \varepsilon, \nu} g_{\varepsilon \beta}\right)\right) .
\end{aligned}
$$

Putting eq.(7.2) into eq.(7.1), we deduce

$$
\frac{1}{i}\left[\hat{g}_{\mu^{\prime} \nu^{\prime}}(\mathbf{x}, t), \hat{\pi}^{(3) \mu \nu}\left(\mathbf{x}^{\prime}, t^{\prime}\right)\right]_{t=t^{\prime}}=\frac{1}{i}\left[\hat{g}_{\mu^{\prime} \nu^{\prime}}(\mathbf{x}, t), \hat{\pi}^{(2) \mu \nu}\left(\mathbf{x}^{\prime}, t^{\prime}\right)\right.
$$$$
]_{t=t^{\prime}}+\int d \mathbf{y}\left(\frac { \partial \hat { g } _ { \mu ^ { \prime } \nu ^ { \prime } } ( \mathbf { x } , t ) } { \partial \hat { g } _ { \rho \sigma } ( y ) } \frac { \partial } { \partial ( \kappa \frac { - 3 } { 2 } g ^ { \rho \sigma , t } ) } \kappa \left(\frac{1}{2} g^{\mu \nu}\left(-g^{\beta \varepsilon, t} g_{\varepsilon \beta}\right)\right.\right.
$$$$
\left.\left.-\frac{1}{4} g^{t \nu}\left(-g^{\beta \varepsilon, \mu} g_{\varepsilon \beta}\right)-\frac{3}{4} g^{t \mu}\left(-g^{\beta \varepsilon, \nu} g_{\varepsilon \beta}\right)\right)\right)_{p b, t=t^{\prime}=t_{y}}
$$$$
=\frac{1}{i}\left[\hat{g}_{\mu^{\prime} \nu^{\prime}}(\mathbf{x}, t), \hat{\pi}^{(2) \mu \nu}\left(\mathbf{x}^{\prime}, t^{\prime}\right)\right]_{t=t^{\prime}}+\int d \mathbf{y}\left(\delta_{\mu^{\prime}}^{\rho} \delta_{\nu^{\prime}}^{\sigma} \delta(\mathbf{x}-\mathbf{y})\right.
$$

$$
\left(\frac{2}{3} g^{\mu \nu}\left(\delta_{\rho}^{\beta} \delta_{\sigma}^{\varepsilon} \delta_{t}^{t} g_{\varepsilon \beta}\right)+\frac{1}{6} g^{t \nu}\left(-\delta_{\rho}^{\beta} \delta_{\sigma}^{\varepsilon} \delta_{t}^{\mu} g_{\varepsilon \beta}\right)+\frac{1}{2} g^{t \mu}\left(-\delta_{\rho}^{\beta} \delta_{\sigma}^{\varepsilon} \delta_{t}^{\nu} g_{\varepsilon \beta}\right)\right.
$$$$
)_{p b, t=t^{\prime}=t_{y}}=\frac{1}{i}\left[\hat{g}_{\mu^{\prime} \nu^{\prime}}(\mathbf{x}, t), \hat{\pi}^{(2) \mu \nu}\left(\mathbf{x}^{\prime}, t^{\prime}\right)\right]_{t=t^{\prime}}+\int d \mathbf{y}(\delta(\mathbf{x}-\mathbf{y})
$$$$
\left(\frac{2}{3} g^{\mu \nu} g_{\nu^{\prime} \mu^{\prime}}+\frac{1}{6} g^{\mu \nu}\left(-g_{\nu^{\prime} \mu^{\prime}}\right)+\frac{1}{2} g^{\nu \mu}\left(-g_{\nu^{\prime} \mu^{\prime}}\right)\right)_{p b, t=t^{\prime}=t_{y}} \delta\left(\mathbf{x}^{\prime}-\mathbf{y}\right)
$$$$
=\frac{1}{i}\left[\hat{g}_{\mu^{\prime} \nu^{\prime}}(\mathbf{x}, t), \hat{\pi}^{(2) \mu \nu}\left(\mathbf{x}^{\prime}, t^{\prime}\right)\right]_{t=t^{\prime}}+\left(\delta\left(\mathbf{x}-\mathbf{x}^{\prime}\right) \cdot(0)\right)_{p b, t=t^{\prime}=t_{y}}=
$$$$
-\left(\frac{1}{2} \delta_{\mu^{\prime}}^{\nu} \delta_{\nu^{\prime}}^{\mu}+\frac{7}{6} \delta_{\mu^{\prime}}^{\mu} \delta_{\nu^{\prime}}^{\nu}+g^{\mu \nu} g_{\nu^{\prime} \mu^{\prime}}\right) \delta\left(\mathbf{x}-\mathbf{x}^{\prime}\right)=\Delta_{\mu^{\prime} \nu^{\prime}}^{(3) \mu \nu} \delta\left(\mathbf{x}-\mathbf{x}^{\prime}\right)
$$

It is interesting that eq.(7.3) has nothing to do with $\hat{\pi}^{\prime(3) \mu \nu}\left(\mathbf{x}^{\prime}, t^{\prime}\right)$, and is only relevant to $\hat{\pi}^{(2) \mu \nu}\left(\mathbf{x}^{\prime}, t^{\prime}\right)$, these
Using eq.(7.3) and taking $\left(\mu^{\prime}, \nu^{\prime}\right)=(\mu, \nu)$, we have

$$
\frac{1}{i}\left[\hat{g}_{\mu \nu}(\mathbf{x}, t), \hat{\pi}^{(3) \mu \nu}\left(\mathbf{x}^{\prime}, t^{\prime}\right)\right]_{t=t^{\prime}}=-\left(\frac{1}{2} \delta_{\mu}^{\nu} \delta_{\nu}^{\mu}+\frac{7}{6} \delta_{\mu}^{\mu} \delta_{\nu}^{\nu}+g^{\mu \nu} g_{\nu \mu}\right.
$$

$$
\delta\left(\mathbf{x}-\mathbf{x}^{\prime}\right)=-\left(\frac{\mathbf{2}}{\mathbf{3}}+\mathbf{2 4}\right) \delta\left(\mathbf{x}-\mathbf{x}^{\prime}\right)=\Delta_{\mu \nu}^{(3) \mu \nu} \delta\left(\mathbf{x}-\mathbf{x}^{\prime}\right)
$$

When the repeating indexes don't sum up, eq.(7.4) is

$$
\begin{gathered}
\frac{1}{i}\left[\hat{g}_{\mu \nu}(\mathbf{x}, t), \hat{\pi}^{(3) \mu \nu}\left(\mathbf{x}^{\prime}, t^{\prime}\right)\right]_{t=t^{\prime}}=-\left(\frac{1}{2} \delta_{\mu}^{\nu} \delta_{\nu}^{\mu}+\frac{1}{6}\right. \\
+2) \delta\left(\mathbf{x}-\mathbf{x}^{\prime}\right)=\Delta_{\mu \nu}^{(3) \mu \nu} \delta\left(\mathbf{x}-\mathbf{x}^{\prime}\right)
\end{gathered}
$$

Eqs.(7.3)-(7.5) mean that the cananical momenta of the general metric tensor operators $\hat{g}_{\mu \nu}(\mathbf{x}, t)$ are the total momenta $\hat{\pi}^{(3) \mu \nu}\left(\mathbf{x}^{\prime}, t^{\prime}\right)$, the different coefficients $-\left(\frac{1}{2} \delta_{\mu}^{\nu} \delta_{\nu}^{\mu}+\frac{7}{6} \delta_{\mu}^{\mu} \delta_{\nu}^{\nu}+g^{\mu \nu} g_{\nu \mu}\right)$ show their different commutation relations.

\section{QUANTUM GRAVITY OF THE UNIVERSE AND GENERAL BLACK HOLE}

We now give applications of the general theory of quantum gravity to gravitational fields of both the Universe and general black hole, namely, give quantum gravity of the Universe and general black hole.

For the key FRW metrics in current cosmology [58]

$$
d s^{2}=d t^{2}-R(t)\left[\frac{d r^{2}}{1-\kappa r^{2}}+r^{2} d \theta^{2}+r^{2} \sin ^{2} \theta d \varphi^{2}\right.
$$

where $R(t)$ is the scale factor of the universe and $\kappa=$ $1,0,-1$, which correspond to closed universe, flat universe and negative curvature universe respectively. Using eqs.(7.3) and (8.1), we have

$$
\begin{gathered}
\frac{1}{i}\left[\hat{g}_{00}(\mathbf{x}, t), \hat{\pi}^{T 00}\left(\mathbf{x}^{\prime}, t^{\prime}\right)\right]_{t=t^{\prime}}=-\left(\frac{1}{2} \delta_{0}^{0} \delta_{0}^{0}+\frac{7}{6} \delta_{0}^{0} \delta_{0}^{0}+\right. \\
\left.g^{00} g_{00}\right) \delta\left(\mathbf{x}-\mathbf{x}^{\prime}\right)=-\frac{\mathbf{8}}{\mathbf{3}} \delta\left(\mathbf{x}-\mathbf{x}^{\prime}\right)=\Delta_{00}^{T 00} \delta\left(\mathbf{x}-\mathbf{x}^{\prime}\right) \\
\frac{1}{i}\left[\hat{g}_{r r}(\mathbf{x}, t), \hat{\pi}^{T r r}\left(\mathbf{x}^{\prime}, t^{\prime}\right)\right]_{t=t^{\prime}}=-\frac{\mathbf{8}}{\mathbf{3}} \delta\left(\mathbf{x}-\mathbf{x}^{\prime}\right)=\Delta_{r r}^{T r r} \delta\left(\mathbf{x}-\mathbf{x}^{\prime}\right)
\end{gathered}
$$


$\frac{1}{i}\left[\hat{g}_{\theta \theta}(\mathbf{x}, t), \hat{\pi}^{T \theta \theta}\left(\mathbf{x}^{\prime}, t^{\prime}\right)\right]_{t=t^{\prime}}=-\frac{\mathbf{8}}{\mathbf{3}} \delta\left(\mathbf{x}-\mathbf{x}^{\prime}\right)=\Delta_{\theta \theta}^{T \theta \theta} \delta\left(\mathbf{x}-\mathbf{x}^{\prime}\right) . \quad \frac{1}{i}\left[\hat{g}_{\varphi \varphi}(\mathbf{x}, t), \hat{\pi}^{T \varphi \varphi}\left(\mathbf{x}^{\prime}, t^{\prime}\right)\right]_{t=t^{\prime}}=-\left(\frac{\mathbf{5}}{\mathbf{3}}+\right.$

$\left.\frac{1}{i}\left[\hat{g}_{\varphi \varphi}(\mathbf{x}, t), \hat{\pi}^{T \varphi \varphi}\left(\mathbf{x}^{\prime}, t^{\prime}\right)\right]_{t=t^{\prime}}=-\frac{\mathbf{8}}{\mathbf{3}} \delta\left(\mathbf{x}-\mathbf{x}^{\prime}\right)=\Delta_{\varphi \varphi}^{T \varphi \varphi} \delta\left(\mathbf{x}-\mathbf{x}^{\prime}\right) \cdot \frac{\mathbf{g}_{\mathbf{0 0}} \mathbf{g}_{\varphi \varphi}}{\mathbf{g}_{\mathbf{0 0}} \mathbf{g}_{\varphi \varphi}-\mathbf{g}_{\mathbf{0} \varphi}^{\mathbf{2}}}\right) \delta\left(\mathbf{x}-\mathbf{x}^{\prime}\right)=\Delta_{\varphi \varphi}^{T \varphi \varphi} \delta\left(\mathbf{x}-\mathbf{x}^{\prime}\right)$.

where $T$ is the total in the canonical momentum fields. The other commutation relations of gravitational tensor fields $\hat{g}_{\mu^{\prime} \nu^{\prime}}(\mathbf{x}, t)$ and thier canonical total momentum fields $\hat{\pi}^{T \mu \nu}\left(\mathbf{x}^{\prime}, t^{\prime}\right)$ are zero, which and eqs.(8.2)-(8.5) just show the homogeneity and isotropy of the Universe.

For the gravitatiional fields of the most general black hole, i.e., the metrics of Kerr-Newman black hole [58]

$$
\begin{aligned}
d s^{2}=( & \left.1-\frac{2 m r-Q^{2}}{r^{2}+a^{2} \cos ^{2} \theta}\right) d t^{2}-\frac{r^{2}+a^{2} \cos ^{2} \theta}{r^{2}+a^{2}-2 m r+Q^{2}} d r^{2} \\
& -\left(r^{2}+a^{2} \cos ^{2} \theta\right) d \theta^{2}-\left[\left(r^{2}+a^{2}\right) \sin ^{2} \theta+\right.
\end{aligned}
$$

$$
\delta\left(\mathbf{x}-\mathbf{x}^{\prime}\right)=\Delta_{0 \varphi}^{T 0 \varphi} \delta\left(\mathbf{x}-\mathbf{x}^{\prime}\right)=\boldsymbol{\Delta}_{\varphi \mathbf{0}}^{\mathbf{T} \varphi \mathbf{0}} \delta\left(\mathbf{x}-\mathbf{x}^{\prime}\right)
$$

The other commutation relations of gravitational tensor fields $\hat{g}_{\mu^{\prime} \nu^{\prime}}(\mathbf{x}, t)$ and their canonical total momentum fields $\hat{\pi}^{T \mu \nu}\left(\mathbf{x}^{\prime}, t^{\prime}\right)$ are zero, which and eqs.(8.9) and (8.10) just show the symmetric properties of Kerr-Newman $\left.\frac{\left(2 m r-Q^{2}\right) a^{2} \sin ^{4} \theta}{r^{2}+a^{2} \cos ^{2} \theta}\right] d \varphi^{2}+\frac{\left(2 m r-Q^{2}\right) a \sin ^{2} \theta}{r^{2}+a^{2} \cos ^{2} \theta}(d t d \varphi+d \varphi d t)$

then, eq.(8.6) can be equivalently and simply expressed as

$$
\begin{gathered}
g_{\mu \nu}=\left(\begin{array}{cccc}
g_{00} & 0 & 0 & g_{0 \varphi} \\
0 & g_{r r} & 0 & 0 \\
0 & 0 & g_{\theta \theta} & 0 \\
g_{\varphi 0} & 0 & 0 & g_{\varphi \varphi}
\end{array}\right) \longrightarrow \\
g^{\mu \nu}=\left(\begin{array}{cccc}
\frac{g_{\varphi \varphi}}{g_{00} g_{\varphi \varphi}-g_{0 \varphi}^{2}} & 0 & 0 & \frac{-g_{\varphi 0}}{g_{00} g_{\varphi \varphi}-g_{0 \varphi}^{2}} \\
0 & \frac{1}{g_{r r}} & 0 & 0 \\
0 & 0 & \frac{1}{g_{\theta \theta}} & 0 \\
\frac{-g_{0 \varphi}}{g_{00} g_{\varphi \varphi}-g_{0 \varphi}^{2}} & 0 & 0 & \frac{g_{00}}{g_{00} g_{\varphi \varphi}-g_{0 \varphi}^{2}}
\end{array}\right)
\end{gathered}
$$

using eqs.(7.3), (8.6) and (8.7), we achieve

$\frac{1}{i}\left[\hat{g}_{00}(\mathbf{x}, t), \hat{\pi}^{T 00}\left(\mathbf{x}^{\prime}, t^{\prime}\right)\right]_{t=t^{\prime}}=-\left(\frac{1}{2} \delta_{0}^{0} \delta_{0}^{0}+\frac{7}{6} \delta_{0}^{0} \delta_{0}^{0}+g^{00} g_{00}\right) \delta(\mathbf{x}-$ black hole about diagonal elements in the covariant metrics of eq.(8.7), eqs.(8.8), (8.11) and (8.12) just further show the effects of the asymmetric property produced from the nondiagonal element field $\hat{g}_{0 \varphi}$ in the covariant metrics of eq.(8.7) for Kerr-Newman black hole.

\section{SUMMARY AND CONCLUSION}

This paper studies the puzzles of quantum gravity, gives a novel general theory of quantum gravity, no relevant to any concrete metric models, shows the solutions to the puzzles, further gives applications of the general theory of quantum gravity to gravitational fields of both the Universe and general black hole, namely, give quantum gravity of the Universe and general black hole.

This paper deduces general commutation relations of the general gravitational field operators and their different styles of high order canonical momentum operators for this general nonlinear system of the standard gravitational Lagrangian.

This paper concretely show the general commutation relations of the general gravitational field operators and their zeroth, first, second and third style, respectively, of

$\left.\mathbf{x}^{\prime}\right)=-\left(\frac{\mathbf{1}}{\mathbf{2}}+\frac{\mathbf{7}}{\mathbf{6}}+\frac{\mathbf{g}_{\varphi \varphi} \mathbf{g}_{\mathbf{0 0}}}{\mathbf{g}_{\mathbf{0 0}} \mathbf{g}_{\varphi \varphi}-\mathbf{g}_{\mathbf{0} \varphi}^{\mathbf{2}}}\right) \delta\left(\mathbf{x}-\mathbf{x}^{\prime}\right)=\boldsymbol{\Delta}_{\mathbf{0 0}}^{\mathbf{T 0 0}} \delta\left(\mathbf{x}-\mathbf{x}^{\prime}\right)$ high order canonical mementum operators for this general nonlinear system of the standard gravitatioal Lagrangian, and then have finished all the four styles of quantization of the standard gravity. Especially, the novel equations (6.4) and (6.5) are deduced for the first time, which reflect the nonlinear structure properties of the commutation relations for the standard gravitatioal Lagrangian, i.e., this paper discovers $\Delta_{\mu \nu}^{(i) \alpha \beta}(i=0,1,2,3)$ $\frac{1}{i}\left[\hat{g}_{r r}(\mathbf{x}, t), \hat{\pi}^{T r r}\left(\mathbf{x}^{\prime}, t^{\prime}\right)\right]_{t=t^{\prime}}=-\frac{\mathbf{8}}{\mathbf{3}} \delta\left(\mathbf{x}-\mathbf{x}^{\prime}\right)=\Delta_{r r}^{T r r} \delta\left(\mathbf{x}-\mathbf{x}^{\prime}\right)$.

$\frac{1}{i}\left[\hat{g}_{\theta \theta}(\mathbf{x}, t), \hat{\pi}^{T \theta \theta}\left(\mathbf{x}^{\prime}, t^{\prime}\right)\right]_{t=t^{\prime}}=-\frac{\mathbf{8}}{\mathbf{3}} \delta\left(\mathbf{x}-\mathbf{x}^{\prime}\right)=\Delta_{\theta \theta}^{T \theta \theta} \delta\left(\mathbf{x}-\mathbf{x}^{\prime}\right)$. and their relations reflecting symmetric propertis of the standard nonlinear gravitational Lagrangian. 
In this paper, we present a new quantization method of gravitation fields of the standard gravitational Lagrangian: the general operator expression of the gravitational field is taken, further the canonical momenta of the gravitational fields are given out by the Lagrangian.

Therefore, people, no as usual, really needn't to solve the Euler-Lagrange equation to fulfill the whole process of the quantization of the standard gravitational fields. Because there also are the unsolvable cases, but this paper shows exact solutions. So a new simple and effective quantization method of gravitational field is presented. So much of the complex calculations of quantum gravitational field theory up to now can be omitted to make the physical picture clearer and simpler, namely, this paper novelly simplifies all the current quantization theories of the standard gravitational field Lagrangian, i.e., novelly gives the quantum gravitational field theory, which make the quantum gravitational field theory more easily understanding and the length shorter of the textbook with key physics formulism and so on. Since quantum gravitational field theory is the very important foundational theory for studying different gravitational field theories. Finally, the conclusions are completely consistent with the existing quantum gravitational field theories. The other processings for the quantization are similar to the usual quantization processings, thus we don't repeat here.

So far as is known to all, the ultimate form of quantum gravity is what all people don't know. This paper presents a general theory of quantum gravity that does not depend on the metric, which provides a general theory for finding the final theory of quantum gravity. Because the quantum gravity theory without dependence of the metric in this paper is the most general, which meet the requirements of the ultimate quantum gravity.

Therefore, this paper opens a door to study and give a simpler, direct physical and easily understandable novel general theory of the quantum gravitational theory don't depending on any concrete metric models. Following this paper, lots of works can be done.

Acknowledgments: The work is supported by the U.S. Department of Energy, contract no. DE-AC0205CH11231, NSF through grants PHY-08059, DOE through grant DEFG02- 91ER40681 and National Natural Science Foundation of China (No. 11875081).

* Electronic address: c.huang0@hotmail.com

$\dagger$ Electronic address: ychuang@bjut.edu.cn

¥ Electronic address: xfli@bjut.edu.cn

[1] Carlo Rovelli, "Quantum gravity". Scholarpedia. 3 (5) (2008) 7117.

[2] David J. Griffiths, Introduction to Quantum Mechanics. Pearson Prentice Hall, (2004).

[3] Robert M. Wald, General Relativity. University of Chicago Press, (1984), p. 382.
[4] Feynman, Richard P.; Morinigo, Fernando B.; Wagner, William G. (1995). Feynman Lectures on Gravitation. Reading, Mass.: Addison-Wesley.

[5] Gary Shiu, Bret Underwood, Kathryn M. Zurek, and Devin G. E. Walker, Probing the Geometry of Warped String Compactifications at the Large Hadron Collider, Phys. Rev. Lett. 100, 031601 (2008); Gary Shiu, Pablo Soler, and Fang Ye, Millicharged Dark Matter in Quantum Gravity and String Theory, Phys. Rev. Lett. 110, 241304 (2013).

[6] Yong-Chang Huang, L. Liao and X. G. Lee, FaddeevJackiw canonical path integral quantization for a general scenario, its proper vertices and generating functionals, The European Physical Journal, C60 (2009) 481-487.

[7] Anthony Zee, Quantum Field Theory in a Nutshell (2nd ed.). Princeton Univ. Press, (2010), p172, 434-435.

[8] Roger Penrose, The road to reality : a complete guide to the laws of the universe. Vintage, (2007), p. 1017.

[9] Bryce S. DeWitt, Giampiero Espositoc, An introduction to quantum gravity, Int. J. Geom. Meth. Mod. Phys.5:101-156,2008.

[10] J. R. Klauder, gr-qc/0612168.

[11] R. Geroch, Ann. Phys. (N.Y.) 62, 582 (1971).

[12] A. Ashtekar, Phys. Rev. Lett. 46, 573 (1981).

[13] B. S. DeWitt, Phys. Rev. 160, 1113 (1967).

[14] C. J. Isham and A. C. Kakas, Class. Quantum Grav. 1, 621 (1984).

[15] A. Ashtekar, Phys. Rev. Lett. 57, 2244 (1986).

[16] G. Esposito, G. Gionti and C. Stornaiolo, Nuovo Cim. B110, 1137 (1995).

[17] G. E. Volovik, The Universe in a Helium Droplet, Int. Ser. Monogr. Phys. 117, 1 (2006).

[18] C. W. Misner, Rev. Mod. Phys. 29, 497 (1957).

[19] B. S. DeWitt, Phys. Rev. 162, 1195 (1967).

[20] G. 't Hooft and M. Veltman, Ann. Inst. H. Poincare, A20, 69 (1974).

[21] M. H. Goroff and A. Sagnotti, Nucl. Phys. B266, 709 (1986).

[22] I. G. Avramidi, Nucl. Phys. B355, 712 (1991).

[23] G. A. Vilkovisky, Class. Quantum Grav. 9, 895 (1992).

[24] F. Canfora, Nucl. Phys. B731, 389 (2005).

[25] G. W. Gibbons and S.W. Hawking, Phys. Rev. D15, 2752 (1977).

[26] S. W. Hawking, Phys. Rev. D18, 1747 (1978).

[27] P. Menotti and A. Pelissetto, Phys. Rev. D35, 1194 (1987).

[28] M. Carfora and A. Marzuoli, Class. Quantum Grav. 9, 595 (1992).

[29] C. Rovelli and L. Smolin, Phys. Rev. Lett. 61, 1155 (1988).

[30] C. Rovelli and L. Smolin, Nucl. Phys. B331, 80 (1990).

[31] J. Gracia-Bondia, hep-th/0206006.

[32] C. J. Isham, Class. Quantum Grav. 6, 1509 (1989).

[33] J. A. Wheeler, Ann. Phys. (N.Y.) 2, 604 (1957).

[34] M. Reuter, Phys. Rev. D57, 971 (1998).

[35] O. Lauscher and M. Reuter, hep-th/0511260.

[36] K. Stelle, Phys. Rev. D16, 953 (1977).

[37] E. Witten, hep-th/0212247.

[38] G. T. Horowitz, New J. Phys. 7, 201 (2005).

[39] A. O. Barvinsky, Phys. Rev. D74, 084033 (2006).

[40] Yong-Chang Huang and C. X. Yu, Quantization and spectrum of open 2-brane, Phys. Rev. D 75 (2007)044011.

[41] D. Z. Freedman, P. van Nieuwenhuizen and S. Ferrara, Phys. Rev. D13, 3214 (1976). 
[42] P. van Nieuwenhuizen, Phys. Rep. 68, 189 (1981).

[43] G. Gionti, gr-qc/9812080.

[44] J. Ambjorn, M. Carfora, D. Gabrielli, A. Marzuoli, Nucl. Phys. B542, 349 (1999).

[45] R. Loll, arXiv:0711.0273.

[46] A. Kheyfets, N.J. Lafave, W.A. Miller, Class. Quantum Grav. 6, 659 (1989).

[47] R. Penrose and M.A.H. MacCallum, Phys. Rep. 6, 241 (1972).

[48] R. Penrose, Class. Quantum Grav. 16, A113 (1999).

[49] Bose, S.; et al. (2017). "Spin Entanglement Witness for Quantum Gravity". Physical Review Letters. 119 (4): 240401.

[50] Marletto, C.; Vedral, V. (2017). "Gravitationally Induced Entanglement between Two Massive Particles is Sufficient Evidence of Quantum Effects in Gravity". Physical Review Letters. 119 (24): 240402.

[51] Nemirovsky, J.; Cohen, E.; Kaminer, I. (30 Dec 2018). "Spin Spacetime Censorship". arXiv:1812.11450v1.

[52] K. Becker, M. Becker, John H. Schwarz, String Theory and M-Theory, Cambridge University Press, 2006.

[53] Wheeler, John Archibald (2010). Geons, Black Holes, and Quantum Foam: A Life in Physics. W. W. Norton \&
Company. p. 235. ISBN 9780393079487.

[54] Wald, Robert M. (1994). Quantum Field Theory in Curved Spacetime and Black Hole Thermodynamics. University of Chicago Press.

[55] S. W. Hawking and R. Penrose, Proc. R. Soc. Lond. A314, 529 (1970).

[56] C. Huang, Yong-Chang Huang, Bao-Hua Zhou, SU(2) gauge field theories, gauge-invariant angular momenta, and a Coulomb theorem: A new viewpoint on the resolution of the nucleon spin crisis, PHYSICAL REVIEW D 92 ( 2015 ) 056003; X. Zhai, C. Huang \& Gang Ren, Extended harmonic mapping connects the equations in classical, statistical, fluid, quantum physics and general relativity, Scientific Reports, 10 (2020 ) 1-12.

[57] C. Huang, Yifei He, Martin Kruczenski, Minimal area surfaces dual to Wilson loops and the Mathieu equation, JOURNAL OF HIGH ENERGY PHYSICS, 8 ( 2016) 088.

[58] Jerzy Plebański and Andrzej Krasiński, An introduction to general relativity and cosmology, Cambridge University Press, 2006. 